\title{
Secondary Organic Aerosol Formation Enhanced by Organic Seeds of Similar Polarity at Atmospherically Relative Humidity
}

\section{AUTHORS: Catherine A. Gordon, Jianhuai Ye, and Arthur W.H. Chan \\ SCHOOL: Department of Chemical Engineering and Applied Chemistry, University of Toronto}

\section{Abstract}

Secondary Organic Aerosol (SOA) forms in the atmosphere when semi-volatile oxidation products from biogenic and anthropogenic hydrocarbons condense onto atmospheric particulate matter. Climate models assume that oxidation products and preexisting organic aerosol form a well-mixed particle and enhance condensation, and, as a result, predict that future increases in anthropogenic primary organic aerosol (POA) will cause a significant increase in SOA. However, recent experiments performed at low humidity $(<10 \%)$ demonstrate a single-phase particle does not always form, challenging the validity of model assumptions. In this work, we investigate the formation of SOA at atmospherically relevant humidities (55-65\%) and examine this mixing assumption. We hypothesized that humidity leads to decreased viscosity and shorter mixing timescales, which is favorable for aerosol mixing. Here, $\alpha$-pinene, a biogenic volatile organic compound is oxidized with ozone in a flow tube reactor in the presence of different organic aerosol seeds. Increased humidity did not enhance SOA formation with erythritol or squalane seed as hypothesized, implying that these compounds do not mix with $\alpha$-pinene SOA in the range of humidities studied (55$65 \%$ ). Yield enhancements were observed with tetraethylene glycol seed, demonstrating interaction between the SOA and seed. These observations suggest increased humidity does not promote mixing between the oxidation products and POA and highlight the need to fully understand the aerosol phase state in the atmosphere in order to better parameterize SOA formation and accurately predict future changes in air quality.

\section{Key Words}

Secondary Organic Aerosol, Atmospheric Chemistry, $\alpha$-pinene, Atmospheric Oxidation, Biogenic-Anthropogenic Interactions

\section{Introduction}

Secondary Organic Aerosol (SOA) plays a significant role in global climate change and poses serious health effects. Oxidation of volatile organic compounds (VOC) emitted from vegetation and human activities leads to low-volatility products, which condense and form SOA (1). Traditional views of SOA formation mechanisms are based on gas-particle partitioning theory: SOA is assumed to mix well with preexisting organic aerosol to form a homogeneous liquid phase (2). By Raoults' Law, the lowering of liquid phase activities leads to greater condensation of semivolatile compounds and higher SOA yields. As a consequence of this well-mixed assumption and predictions of higher future anthropogenic emissions, climate models are predicting a $10-30 \%$ increase in SOA levels (3-6). This mechanism of increasing SOA formation is currently being challenged, as laboratory experiments have demonstrated that a wellmixed particle does not always form (7-9). However, most of these experiments were conducted under dry conditions, (with relative humidities, $\mathrm{RH}$, less than $20 \%(10)$ ), and there has been no comprehensive study performed at atmospherically relevant humidities (RH between 40 and 90\%). This poses two problems: 1) low humidity is not necessarily representative of true atmospheric conditions, and 2) the absence of significant water may be impacting the interaction between SOA and other atmospheric particulate matter.

In this study, we investigate the mixing between SOA and a variety of preexisting organic aerosols under atmospherically relevant humidity conditions. By increasing the humidity and therefore the water content present, particle viscosity may decrease, resulting in shorter aerosol mixing timescales. We hypothesize that under atmospherically relevant conditions ( $55-65 \%$ relative humidity), enhanced SOA yields may be observed. Squalane, tetraethylene glycol and erythritol were chosen as organic seeds based on their polarities and relevance to atmospheric organic compounds.

\section{Materials and Methods}

In this work, we study the oxidation of $\alpha$-pinene, an important biogenic VOC, by ozone in laboratory experiments. Experiments were conducted in a quartz flow tube with a volume of $10 \mathrm{~L}$. The total flow rate through the flow tube was maintained at $2.5 \mathrm{~L} \mathrm{~min}^{-1}$, giving the flow tube a residence time of 4 minutes. To perform $\alpha$-pinene ozonolysis experiments, $\alpha$-pinene, water vapour, seed particles, and ozone were separately introduced into the flow tube and the flow rates were controlled using mass flow controllers (MKS).

To achieve 55-65\% RH, two humidifiers, connected in series, were used to introduce water vapour. Each humidifier consisted of a sealed container of milliQ water $(18.2 \mathrm{M} \Omega / \mathrm{cm})$ with a bubbler at the inlet, through which purified air flowed. Humidity and temperature 
were monitored throughout the experiment using an Omega HX94C $\mathrm{RH} / \mathrm{T}$ transmitter. A syringe pump (KDS Legato 100) was used to inject $\alpha$-pinene (Sigma-Aldrich, 98\% pure) in a 1:100 solution with cyclohexane (Sigma-Aldrich, 99.5\% pure) from a $1000 \mu \mathrm{L}$ syringe (Hamilton). The syringe pump continuously injected $\alpha$-pinene solution into the humidified dilution air, which flowed into the flow tube. Ozone was separately added to the flow tube by passing purified air through an ozone generator (UVP).

The seed aerosols chosen for this experiment were ammonium sulphate, erythritol, tetraethylene glycol (TEG), and squalane. Ammonium sulphate aerosol, an inorganic particle that is not expected to interact with $\alpha$-pinene SOA, acts as a baseline for comparison. Erythritol, ammonium sulphate and TEG were atomized in an aqueous or methanol solution using a TSI 3076 Aerosol Generator. Squalane seed was generated through homogeneous nucleation (11). Purified air was passed over liquid squalane heated to $\sim 129^{\circ} \mathrm{C}$. Squalane particles were formed by passing the squalane vapor stream through a water-cooled condenser. A custom-made diffusion dryer was used to dry seed aerosols, and a honey-comb charcoal denuder was used for methanol removal before seed aerosol entered the flow tube.

The $\alpha$-pinene consumption was monitored using Gas Chromatography - Flame Ionization Detection (GC-FID, SRI 8610C) equipped with a trap (Tenax TA), which collected gas samples at a flow rate of $\sim 0.12 \mathrm{~L} \mathrm{~min}^{-1}$ for 5 minutes before analysis. Particle volume concentration was measured using a Scanning Mobility Particle Sizer (SMPS, TSI 3081 classifier and TSI 3772 condensation particle counter) and converted to mass concentration with a density of $1.20 \mathrm{~g} \mathrm{~cm}^{-3}$. SOA yields were calculated as the mass of SOA generated per mass of $\alpha$-pinene consumed, shown as follows:

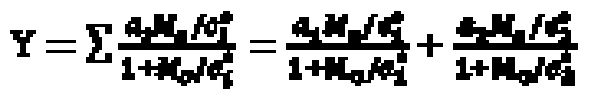

Where $\triangle \mathrm{SOA}$ is the increase of SOA mass concentration and $\triangle H C$ is the change in $\alpha$-pinene mass concentration. Yield curves were fit using Volatility Basis Set Model (12). This semi-empirical model describes the absorptive partitioning between gas and particle phases of semivolatile organic compounds by grouping them into prescribed volatility bins. In this study, $10 \mu \mathrm{g} \mathrm{m}^{-3}$ and $100 \mu \mathrm{g}$ $\mathrm{m}^{-3}$, denoted as $\mathrm{c}_{1}{ }^{*}$ and $\mathrm{C}_{2}{ }^{*}$, respectively, are used as the two volatility bins of interest for atmospherically relevant organic aerosol concentrations, Mo.
Yield curves can ho fittod ucins tho follomsins Equation:

$$
\text { Yiald }=Y=\frac{\triangle S O A}{\triangle H C}
$$

\section{Results}

Figure 1 shows a SOA yield curve (SOA yield as a function of generated SOA mass concentration) with AS seed. According to the optical and fluorescence image results from You et al., $\alpha$-pinene SOA forms a separated phase from AS in the atmosphere even at relative humidities as high as $70 \%$ (13). This yield curve therefore represents the partitioning of SOA into a separate particle phase which has no interaction with the AS seed. In this study, this yield curve with AS seed is used as a baseline against which yield curves from $\alpha$-pinene SOA and other seeds are compared: SOA yields similar to those with AS seed indicate that the seed material does not interact with $\alpha$-pinene SOA; SOA yields enhanced above the AS curve indicate the interaction between seeds and $\alpha$-pinene SOA. In our experiments, organic seeds range in polarity from hydrophobic (squalane) to moderately hydrophilic (TEG) to highly water-soluble (erythitol).

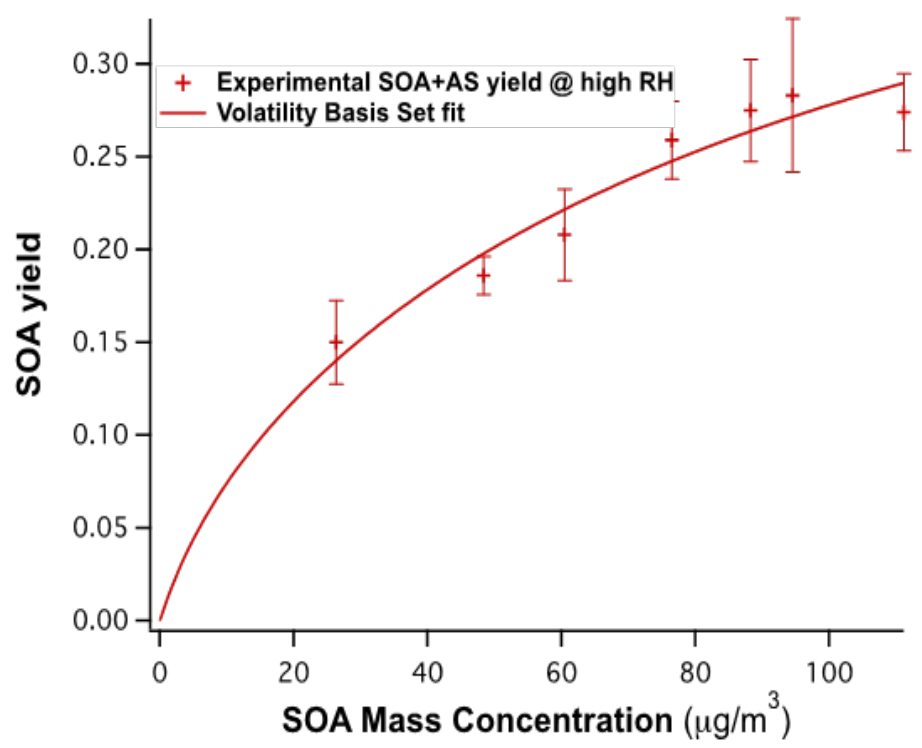

Figure 1: SOA yield formed from $\alpha$-pinene ozonolysis as a function of SOA mass concentration at $55-65 \%$ relative humidity in presence of ammonium sulphate seed particles.

The error bars represent the standard deviation of 3-4 GC-FID measurements and 15-20 SMPS measurements.

As shown in Fig. 2, Erythritol and squalane seed showed no evidence of mixing with $\alpha$-pinene SOA. SOA yields for both seed materials are similar to those with AS seed within experimental uncertainties, indicating that no interaction can be observed. 


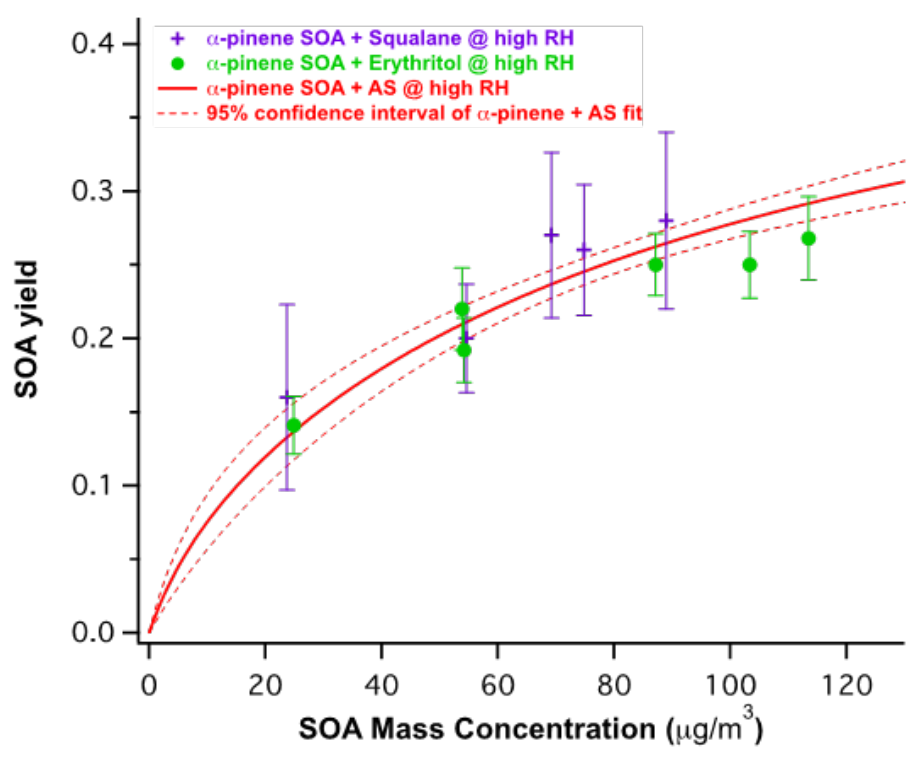

Figure 2: SOA yield formed from $\alpha$-pinene ozonolysis versus SOA generated in presence of ammonium sulfate (red), erythritol (green) and squalane (purple) particles.

The error bars represent the standard deviation of 3-4 GC-FID measurements and 15-20 SMPS measurements.

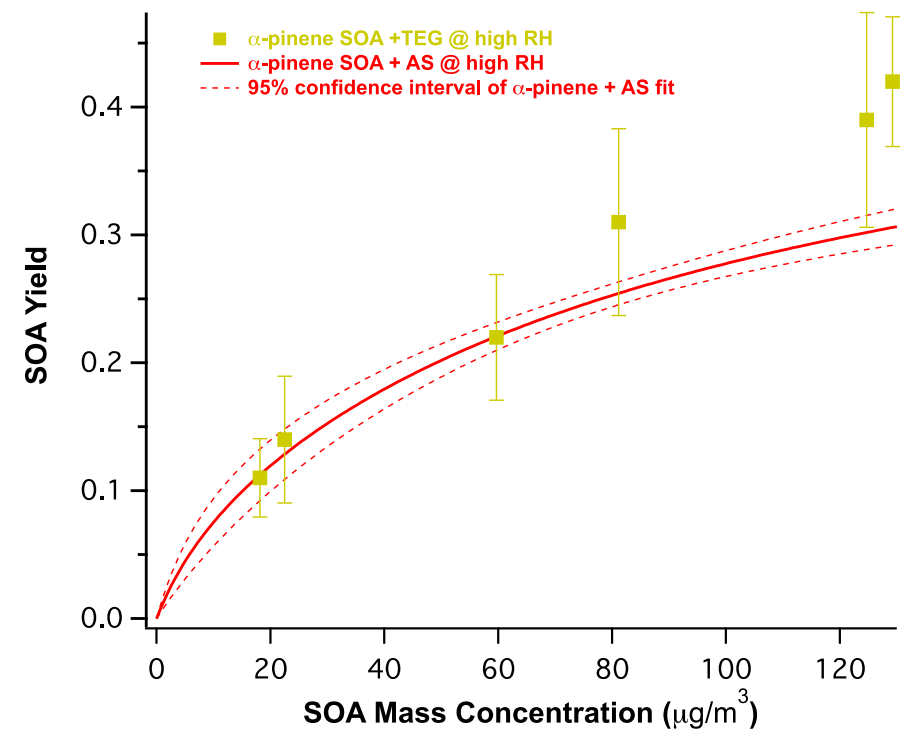

Figure 2: SOA yield formed from $\alpha$-pinene ozonolysis versus SOA generated in presence of ammonium sulfate (red) and tetraethylene glycol (yellow).

The error bars represent the standard deviation of 3-4 GC-FID measurements and 15-20 SMPS measurements.
TEG seed enhanced SOA formation compared to AS, suggesting TEG is miscible with $\alpha$-pinene SOA to some extent, as shown in Figure 3. It is noteworthy that the yield enhancement by TEG occurs at an organic aerosol concentration of $80 \mu \mathrm{g} / \mathrm{m}^{3}$ or higher. $\alpha$-pinene SOA is a collection of particle-phase oxidation products across a range of volatilities. It is therefore likely that TEG mixes with $\alpha$-pinene oxidation products that have an effective saturation concentration of $80 \mu \mathrm{g} / \mathrm{m}^{3}$ or higher, causing more of these compounds to condense than $\alpha$-pinene SOA alone.

\section{Discussion}

These observations (lack of mixing of SOA with squalane and erythritol, and some mixing of SOA with TEG) are consistent with those obtained from analogous low humidity experiments performed in our lab. Here, while increasing the humidity from $10 \%$ to $55-65 \%$ may result in decreased particle phase viscosities, the particle mixing timescales are still beyond the residence time of the flow tube experiments (4 minutes). Contrary to common model assumptions, there is no evidence to show that mixing between SOA and preexisting organic aerosol leads to SOA enhancement at these timescales, even at atmospherically relevant humidities.

Instead, the mixing behavior at these timescales is likely governed by differences in polarities. Using the Hansen Solubility Parameter framework, we can calculate the polarity force of different materials that contribute to organic mixing (14), as shown in Equation (3):

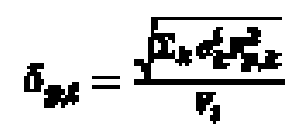

where $V_{i}$ is the molar volume of compound $i, c_{k}{ }^{i}$ the number of the functional groups $\mathrm{k}$ in compound $\mathrm{i}, \mathrm{F}_{\mathrm{p}, \mathrm{k}}^{2}$ is the molar attraction constant for functional group $k$.

The polarity of $\alpha$-pinene SOA is calculated $7.8 \mathrm{MPa}^{1 / 2}$, while TEG, erythritol, and squalane have polarities of $6.2 \mathrm{MPa}^{1 / 2}, 14.2 \mathrm{MPa}^{1 / 2}$ and $0.0 \mathrm{MPa}^{1 / 2}$, respectively. It is evident that the polarity of TEG is most similar to SOA, while erythritol and squalane have significantly different polarities compared to SOA. Here we propose that the mixing propensity of organic aerosol is likely governed by its polarity, as evidenced by the lack of mixing between $\alpha$-pinene SOA (moderately polar) with erythritol (most polar) and squalane (least polar), and mixing with the mildly polar TEG. SOA yield enhancement occurs only when the organic seed has a similar polarity as $\alpha$-pinene SOA. Results from similar experiments performed by Song et al. also show that mixing occurs for some organic seeds only $(7,8)$. 
The mixing behavior from those experiments can also be explained by the seed polarity, since the only seed that led to yield enhancement (citric acid) has a polarity of $7.9 \mathrm{MPa} 1 / 2$, calculated using the above method. As a result, the similarities in mixing behavior between dry and humid experiments, and across different studies, suggest that a framework describing mixing between aerosols of polarities is needed for atmospheric models to accurately predict SOA formation.

\section{Conclusion}

In this work we conducted analysis of SOA formation from $\alpha$-pinene ozonolysis in the presence of different seeds. Organic seeds such as erythritol and squalane demonstrated no interaction with $\alpha$-pinene SOA, but TEG showed evidence of interaction leading to enhancement in SOA yields. We see no evidence that humidity at atmospherically relevant levels (55-65\%) would lead to the formation of well-mixed SOA particles, as originally hypothesized. Our results suggest that even at atmospherically relevant humidities, mixing does not universally occur between biogenic oxidation products and pre-existing aerosols, as is currently assumed by climate change models. There are likely strict mixing criteria for organic aerosols based on polarities, and treating aerosol as a well-mixed liquid phase could lead to over-predictions of SOA formation.
Investigation of a greater variety of seed materials, such as nhexadecanol and citric acid, will allow for further comparison to low humidity results. Additionally, examination of the composition of $\alpha$-pinene SOA formed under high humidity will allow further investigation of mixing behaviors. Performing experiments with longer mixing timescales under humid conditions will allow further investigation of the effect of interaction time on SOA formation.

Our group has developed a model based on compound polarities to predict organic miscibility under dry conditions; the similarities in SOA mixing between dry and humid SOA yields shown here suggest that this framework may be applied to atmospherically relevant humidities. Ultimately, our observations highlight the need to fully understand the aerosol phase state in the atmosphere in order to better parameterize SOA formation and predict future air quality.

\section{Acknowledgements}

The authors would like to acknowledge funding support from NSERC Discovery Grant (435771-13) and University of Toronto Centre for Global Change Science. JY is supported by the Ontario Trillium Scholarship.

\section{References}

1. Hallquist M, Wenger J, Baltensperger U, Rudich Y, Simpson D, Claeys M. The formation, properties and impact of secondary organic aerosol: current and emerging issues. Atmos. Chem. Phys. 2009;9(14):5155-5236.

2. Pankow J. An absorption model of gas/particle partitioning of organic compounds in the atmosphere. Atmos. Env. 1994;28(2):185-188.

3. Liao H, Chen W, Seinfeld J. Role of climate change in global predictions of future tropospheric ozone and aerosols. J Geophys Res. 2006:111(D12304) full DOI:10.1029/2005JD006852.

4. Heald C, Henze D, Horowitz L, Feddema J, Lamarque J, Guenther A. Predicted change in global secondary organic aerosol concentrations in response to future climate, emissions, and land use change. J Geophys Res Atmos. 2008;113(D5): full DOI:10.1029/2007JD009092.

5. Hoyle C, Myhre G, Berntsen T, Isaksen I. Anthropogenic influence on SOA and the resulting radiative forcing. Atmos. Chem. Phys. 2009;9(8):2715-2728.

6. Carlton A, Bhave P, Napelenok S, Edney E, Sarwar G, Pinder R. Model Representation of Secondary Organic Aerosol in CMAQv4.7. Environ. Sci. Technol.. 2010;44(22):8553-8560.

7. Song C, Zaveri R, Alexander M, Thornton J, Madronich S, Ortega J. Effect of hydrophobic primary organic aerosols on secondary organic aerosol formation from ozonolysis of $\alpha$-pinene. Geophys Res Lett. 2007;34(20): full DOI:10.1029/2007GL030720

8. Song C, Zaveri R, Shilling J, Alexander M, Newburn M. Effect of Hydrophilic Organic Seed Aerosols on Secondary Organic Aerosol Formation from Ozonolysis of $\alpha$-Pinene. Environ. Sci. Technol.. 2011;45(17):7323-7329.

9. Asa-Awuku A, Miracolo M, Kroll J, Robinson A, Donahue N. Mixing and phase partitioning of primary and secondary organic aerosols. Geophys Res Lett. 2009;36(15):full DOI: 10.1029/2009GL039301

10. Seinfeld J, Erdakos G, Asher W, Pankow J. Modeling the Formation of Secondary Organic Aerosol (SOA). 2. The Predicted Effects of Relative Humidity on Aerosol Formation in the $\alpha$-Pinene-, $\alpha$-Pinene-, Sabinene-, $\Delta 3$-Carene-, and Cyclohexene-Ozone Systems. Environ. Sci. Technol.. 2001;35(15):3272-3272.

11. Kolesar, K R, Buffaloe G, Wilson K R, Cappa C D. OH-Initiated Heterogeneous Oxidation of Internally-Mixed Squalane and Secondary Organic Aerosol. Environ. Sci. Technol. 2014;48:31963202

12. Donahue N M, Robinson A L, Stanier C O, Pandis S N. A Two-dimensional Volatility Basis Set: 1. Organic-aerosol Mixing Thermodynamics. Environ. Sci. Technol. 2006;40: 2635-2643

13. You Y, Renbaum-Wolff L, Carreras-Sospedra M, Hanna S J, Hiranuma N, Kamal S, et al. Images reveal that atmospheric particles can undergo liquid-liquid phase separations. Proc. Natl. Acad. Sci. USA. 2012;109(33): 13188-13913

14. Compernolle S, Ceulemans K, Muller J F. Influence of non-ideality on condensation to aerosol. Atmos. Chem. Phys. 2009;9:1325-1338 


\section{REVIEW}

\section{by Dr. Darrick V. Heyd,}

\section{Associate Professor and Senior Adviser, Academic Space Planning, Ryerson University}

In the subject manuscript, Gordon et al. report on laboratory-based studies of the miscibility of secondary organic aerosol (SOA) and primary organic aerosol (POA) seed at ambient relative humidity (RH) values. The authors explore two prevalent conventions in atmospheric modeling of SOA formation: 1) SOA and POA are treated as fully miscible in most air quality models; and 2) modeling parameterizations are based on laboratory studies conducted at artificially low RH. As noted by the authors, it is critically important to start conducting laboratory experiments at atmospherically-relevant RH values. Given the paucity of laboratory data at RH values $>10 \%$, the findings in this manuscript will greatly contribute to the communities' understanding of SOA/POA miscibility at higher (closer to ambient) RH values. In addition, these findings contribute to the growing evidence that the modeling conventions described above need revisiting in order to achieve improvements in air quality models. The manuscript is generally well written. It is recommended that minor revisions be made based on the comments below.

\section{Technical Comments:}

Gas/particle partitioning theory does not impose or require mixing as implied in the introduction. It is the application of partitioning theory with the assumption of activity coefficients $=1$ that promotes mixing in a model; when activity coefficients are calculated it has been demonstrated that mixing may not always occur (e.g., see papers by Zeund and Seinfeld; Pankow).

In the discussion of yield enhancements in the TEG case, the authors suggest that the condensing compounds likely have an effective saturation concentration of $80 \mathrm{ug} / \mathrm{m}^{3}$ because that is the SOA concentration at which enhancement occurs. Based on gas/particle partitioning theory, and as articulated in Donahue et al. 2006, when the effective saturation concentration is equal to the $\mathrm{SOA}(/ \mathrm{OA})$ concentration a compound resides $50 \%$ in the gas phase and $50 \%$ in the particle phase. Thus compounds with effective saturation concentrations that are larger than $80 \mathrm{ug} / \mathrm{m}^{3}$ (more volatile) are likely also contributing but have a greater fraction in the gas phase than the particle phase. While the authors do say $80 \mathrm{ug} / \mathrm{m}^{3}$ or higher, I think they mean $80 \mathrm{ug} / \mathrm{m}^{3}$ or lower (less volatile) and should consider revisions based on this comment.

Also, the suggestion that TEG mixes with a subset of alpha-pinene oxidation products based on effective saturation concentration alone would only occur if the enhancement in partitioning is due simply to the increase in the size of the absorbing phase (i.e., existing aerosol mass) into which the alpha-pinene oxidation products can condense. Other reasons for the enhancement could be change in activity coefficients of some of the condensing compounds (more favorable for partitioning) and change in the mean MW of the absorbing phase, which are harder to evaluate.

The Hansen Solubility parameter calculations are an important step in assessing the drivers for enhanced partitioning. The results of the Hansen analysis seem to suggest that activity coefficients (representing "likeness") are very important, which may not be represented by the effective saturation concentration. How were functional groups determined for Hansen Solubility parameters? Maybe you want to include a table of your values for Eq. 3? Or if these are coming from the literature (e.g., ref. 14) that needs to be clearer.

\section{Minor/Editorial Comments:}

Introduction: I don't think that is has been shown that SOA per se poses serious health effects. If I am wrong about that, please include a citation. Most of the time we talk about the health effects of fine PM, of which SOA is a significant fraction and thus is associated with serious health effects.

Materials and Methods: Reference for the density (1.2) assumption?

Check consistency of abbreviations (TEG, AS, etc). 